\title{
A RARE CASE OF BRAIN ABSCESS
}

\author{
Dinesh Kumar', Fazil Thaha², Percival Gilvaz ${ }^{3}$
}

${ }^{1}$ Senior Resident, Department of Neurology, JMMCH, Trichur. ${ }^{2}$ Associate Professor, Department of Neurology, JMMCH, Trichur. 3Professor and HOD, Department of Neurology, JMMCH, Trichur.

\section{ABSTRACT}

Brain abscess is caused by inflammation and collection of infected material coming from local or remote infectious site. The usual age of presentation is 2 to 80 years with males being predominately affected. Incidence in India is $8 \%$ and 1 to $2 \%$ in Western countries. $^{1}$

\section{KEYWORDS}

Brain Abscess, Cladosporium.

HOW TO CITE THIS ARTICLE: Kumar D, Thaha F, Gilvaz P. A rare case of brain abscess. J. Evolution Med. Dent. Sci. 2016;5(63): 4491-4493, DOI: $10.14260 /$ jemds/2016/1023

\section{INTRODUCTION}

Brain abscess by Cladosporium is very rarely reported in literature and is usually highly recurrent with high mortality. Cladosporium mostly cause ring enhancing lesion with perifocal oedema in most of cases. Rare cases present with classical meningeal signs. It is highly neurotropic fungus. The predominant route of infection is inhalation of spores and spread to central nervous system via bloodstream. Here, we present a case of 57-year-old diabetic male with brain abscess caused by Cladosporium bantiana. Patient was managed with surgical excision followed by antifungal drugs; 4 months follow-up MRI has not shown any recurrence.

\section{CASE REPORT}

A 57-year-old diabetic male came with history of excruciating headache for 4 weeks and behavioural changes for 1 week. The headache was sudden in onset, gradually progressive associated with vomiting, not associated with any postural variation and not relieved on taking medication from local hospital. No history of fever associated with headache was found on taking history. There was no history of any neck stiffness. On general physical examination patient was afebrile; pallor, cyanosis or jaundice were not present.

On central nervous system examination higher mental functions were normal, there were no signs of cranial nerve involvement. Tone and power was normal. Deep tendon reflexes were brisk bilaterally and cerebellar signs were positive on right side in right upper limb (Finger nose and dysdiadochokinesia). Plantar was withdrawn bilaterally. Fundus examination showed papilloedema more in right eye compared to left. Routine investigations were normal. CT head was done and showed hypodense lesion with thick irregular wall enhancing over right parieto-occipital region with no perilesional oedema. MRI was done and revealed hyperintense enhancing lesion over right parieto-occipital region with thick irregular walls on T2 and hypointense on T1. Abnormal tissue was removed from brain and histopathology examination of

Financial or Other, Competing Interest: None.

Submission 17-06-2016, Peer Review 21-07-2016,

Acceptance 29-07-2016, Published 08-08-2016.

Corresponding Author:

Dr. Dinesh Kumar,

\#F7/29, Mata Kaulan Marg, Kashmir Avenue,

Amritsar-143001, Punjab.

E-mail: manishksharma27@gmail.com

DOI: $10.14260 /$ jemds/2016/1023 brain tissue was suggestive of fungus Cladosporium with brown pigmented branching and septate hyphae. After review of literature no specific treatment was found for the fungus, but the patient was given a trial of antifungal therapy fluconazole $200 \mathrm{mg}$ orally twice daily and patient was followed up. MRI follow-up was taken and there was mild regression of size of lesion and clinically patient improved. The patient is doing well on antifungal treatment on follow-up.

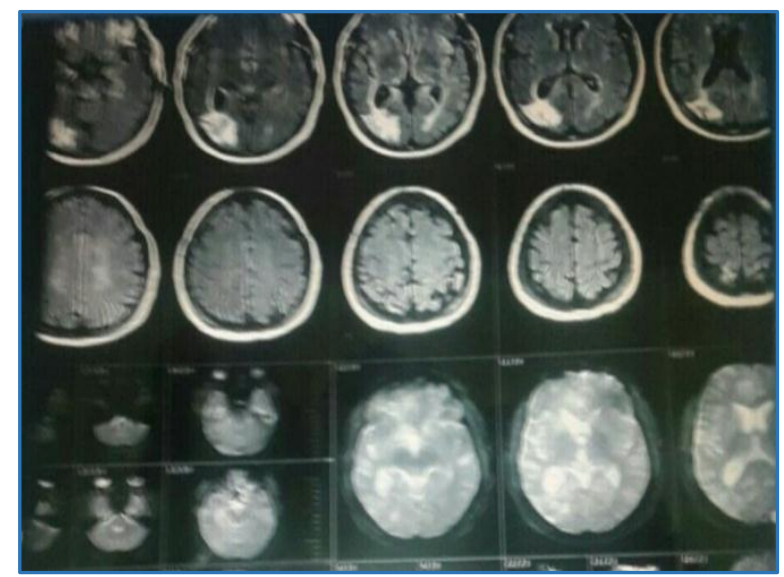

Fig. 1: MRI showing Parieto-Occipital Lesion-Brain Abscess

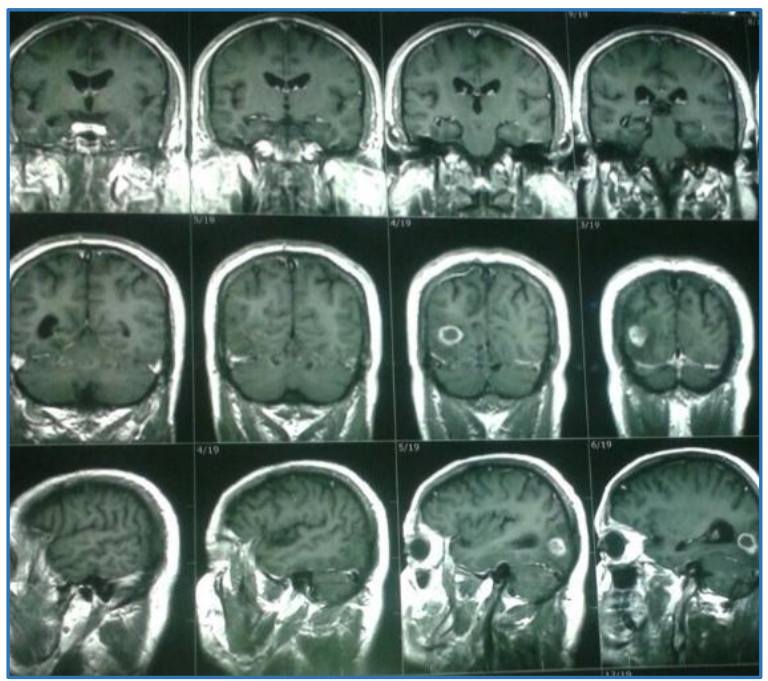

Fig. 2: Coronal and Sagittal MRI - Parieto-Occipital Ring Enhancing Lesion (Brain Abscess) 


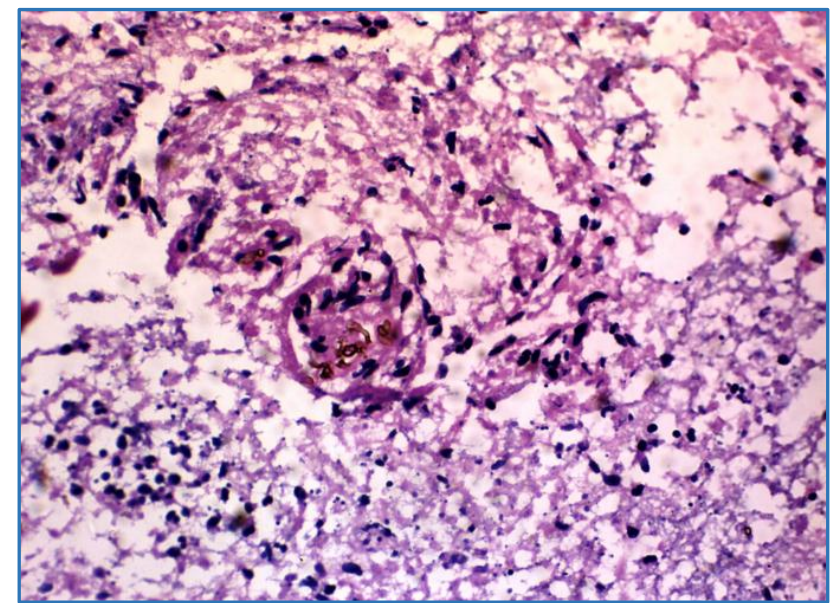

Fig. 3: Haematoxylin and Eosin showing Brown Colonies of Fungal Hyphae

\section{DISCUSSION}

Opportunistic fungal infection is rare and rarely healthy individuals may be affected. The fungus Cladosporium is found in brain and other extracranial sites like lungs, gastrointestinal tract, ear and skin. This fungus has been isolated from soil, bark, detritus, decaying lumber and from brick walls. ${ }^{1}$ It causes deep seated phaeohyphomycosis. The first description of fungus was in 1952. The exact mode of infection is not clear. ${ }^{1}$ This infection can present as two pathomorphological formsdiffuse meningoencephalitis and focal brain abscess. ${ }^{1}$ It has been seen that this fungal infection mostly occurs via inhalation of spores into lungs and subsequent haematogenous dissemination into brain. The most of cases have found to occur in immunocompromised patients following renal transplant and as well as in patients on longterm corticosteroid therapy. The usual clinical features age of occurrence is 6 to 79 years. The onset is more in second and third decade, the male-to-female ratio is $3: 1$. The disease is more common in farmers. Traumatic implantation of fungus due to orbital trauma is also well known. It is a fungus with distinct neurotropism. ${ }^{2}$ Neuroparenchymal lesions caused by it consists of necrotizing granuloma, multifocal in $50 \%$ of cases. $^{2}$

These fungi are becoming increasing opportunistic pathogens in solid organ transplant patients. ${ }^{3}$ The time from transplantation to onset of infection varies from two months to eleven years. These infections are also known to exist in patients of diabetes mellitus and myelodysplastic syndrome. 4 The two clinical forms are cutaneous and cerebral. The fungi is brown-walled fungi with septate round bodies and pigmented branching hyphae. The fungus is stained with methenamine silver and PAS stain. The fungus can grow at thirty seven degrees Celsius on Sabouraud agar for 6 days. The clinical features of patients are usually headache, nausea and vomiting. The clinical presentation can be in forms of seizure, hemiparesis and ataxia. The majority of cases of brain abscess formed by this fungus are multiple. This aggressive mould has poor prognosis related to its poor response to currently available antifungal therapy. 5 The pathophysiology of primary CNS phaeohyphomycosis is unknown. The studies have shown mainly haematogenous route as more likely source of CNS infection from different sites of body, mainly from an initial subclinical pulmonary foci. ${ }^{5}$ The extension of fungus from paranasal sinuses has also been reported. 5
Chronic meningitis without cerebral invasion has also been reported. ${ }^{5}$ The recurrences are common in these fungal brain infections. The complications like ventriculitis and death have been reported in various studies.6,7

The morphology of the infection caused by these fungi may depend on immune status and immune response. 6 Diffuse meningoencephalitis with extensive necrosis has been reported predominately in immunocompromised patients. Lumbar puncture revealed pleocytosis with polymorphonuclear cell predominance in most of patients in various studies. ${ }^{8}$ In absence of contamination, the CSF is generally sterile. The definitive diagnosis can be made at brain autopsy when base of brain grows fungus on culture. The radiological features of CNS cladosporiosis are not characteristic. The CT scan showed the lesion to be either well defined or poorly defined. The well-defined lesions may be single or multiple conglomerate ring-enhancing lesions with perilesional oedema. It is difficult to differentiate this fungal abscess from tubercular or pyogenic abscess. Occasionally, the oedema surrounding may be disproportionate to size of lesions. The abscesses caused by this fungus are usually hemispherical affecting cerebral white matter. Similar CT findings were found in our patient. There are reports of involvement of choroid plexus, thalamus, diencephalons, cerebellum and brainstem in various studies. ${ }^{8}$ The chest radiographs were found to be normal in our patient in this study. The most important factor that determines cure is the pathomorphological form of fungi. The surgical options for treating the fungal lesion include stereotactic biopsy and aspiration, burr hole and tapping and excision. Burr hole and tapping is found inferior to excision. The patients may not have definitive diagnosis on aspiration or may have significant residue requiring multiple attempts on aspiration. The aspirate is also thick preventing adequate decompression. The patients undergoing burr hole and tapping may require craniotomy due to persistence of lesion. The excision in cases with well-defined abscess helps in reducing the oedema, reduction of disease load and better response to antifungal therapy. ${ }^{9}$ It is important to keep patients in close clinical and radiological followup. Once the infection becomes widespread, the outcome is fatal despite aggressive surgical management and antifungal therapy. The efficacy of various antifungal drugs is not very clearly established in humans. Amphotericin B, Itraconazole, Flucytosine, voriconazole and posaconazole have been used with limited success in various studies. ${ }^{10}$ Amphotericin B in combination with other antifungals has been found to have better outcome than monotherapy. Among the various antifungals available for treatment, newer triazoles have shown promising results in animal studies and case reports. The antifungal fluconazole is also found to be effective in some studies. ${ }^{10}$

CNS cladosporiosis is associated with high mortality rate with survival up to 29 to 45 percent. ${ }^{11}$ The outcome in our study is found to be favourable with follow-up treatment on fluconazole.

\section{REFERENCES}

1. Garg N, Devi IB, Vajramni GV, et al. Central nervous system cladosporiosis: an account of ten culture proven cases. NIJ 2007;55(3):282-8. 
2. Deb S, Khan AK, Debashish A, et al. Intracranial necrotizing granuloma caused by cladophialophora bantiana. NIJ 2005;53(3):335-6.

3. Schieffelin JS, Garcia-Diaz JB, Loss GE, et al. Phaeohyphomycosis fungal infections in solid organ transplant recipients: clinical presentation, pathology and treatment. Transpl Infect Dis 2014;16(2):270-8.

4. Huwang WM, Fan YM, Li W, et al. Brain abscess caused by cladophialophora bantiana in China. J Med Microbiol 2011;60(Pt 12):1872-4.

5. Tunuguntia A, Saad MM, Abdalla J, et al. Multiple brain abscesses caused by cladiophialophora bantianum: a challenging case. Tenn Med 2005;98(5):227-8, 235.

6. Dastur HM, Chaukar AP, Rebello MD. Cerebral chromoblastomycosis due to cladosporium trichoides (bantianum) part I. A review and case report. NIJ 1966;14(1):1-5.
7. Desai SC, Bhatikar ML, Mehta RS. Cerebral chromoblastomycosis due to cladosporium trichoides (Bantianum) II. Mycopathologic investigation of brain and skin involvement. NIJ 1966;14:6-18.

8. Dixon DM, Walsh TJ, Merz WG, et al. Infections due to xylohypha bantiana. Cladosporium trichoides. Rev Infect Dis 1989;11(4):515-25.

9. Cooper CR. Deep phaeohyphomycosis. In: Merz WG, Hay RJ, eds. Topley and Wilson's microbiology and microbial infections - medical mycology. $10^{\text {th }}$ edn. London: Hodder Arnold 2005:739-48.

10. Lyons MK, Blair JE, Leslie KO. Successful treatment with voriconazole of fungal cerebral abscesses due to cladophialophora bantiana. Clin Neurol Neurosurg 2005;107(6):532-4.

11. Borges MC, Warren S, White W, et al. Phaeohyphomycosis due to xylohypha bantiana. Arch Pathol Lab Med 1991;115(6):627-9. 